\title{
A seismically constrained mass discharge rate for the initiation of the May 18, 1980 Mount St. Helens eruption
}

\author{
Emily E. Brodsky and Hiroo Kanamori \\ Seismological Laboratory, California Institute of Technology, Pasadena \\ Bradford Sturtevant \\ Graduate Aeronautical Laboratories, California Institute of Technology, Pasadena
}

\begin{abstract}
We calculate the vertical mass discharge rate from Mount St. Helens for the first few minutes of the May 18, 1980 cataclysmic eruption using a new method based on seismic constraints. The observed seismic waves indicate that the seismic source is a series of single forces. We model these forces as thrusts due to a combination of the momentum flux of the erupted products and the pressure of the eruptive jet. The momentum discharge rate is converted to a mass discharge rate based on estimates of the velocity and jet pressure as constrained by a simple fluid dynamical model. Only two parameters are necessary for the calculation: Mach number and sound velocity. The calculated mass ejected in the first $100 \mathrm{~s}$ is $1.6 \times 10^{11}-4.6 \times 10^{11} \mathrm{~kg}$. Since the total blast deposit is $\sim 3.2 \times 10^{11}-4.1 \times 10^{11} \mathrm{~kg}$, one possible interpretation is that the directed blast had a significant $(>30 \%)$ vertical component.
\end{abstract}

\section{Introduction}

One of the most fundamental measures of explosive volcanic eruptions is mass discharge rate. Quantification of the rate and orientation at which mass is ejected improves the scientific understanding of the eruptive process and provides realistic hazard assessment. In this paper, we combine a fluid mechanical model with seismological data to formulate a method for measuring mass emission rate.

The most common previously established method of assessing the volcanic mass emission rate is measurement of the column height. Wilson et al. [1978] show that the height of a plinian column is a function of the energy available for buoyant ascent. Since the energy source is hot mass ejected from the vent, height can be inverted for mass emission rate. This method has proven effective for computing average discharges of plumes [Sparks et al., 1997] but is not applicable for certain eruptive processes including directed blasts, strombolian explosions, and pyroclastic flow generation. In all of these cases the erupted mass of interest does not directly contribute to the buoyant plume and therefore must be measured by other means. Another shortcoming of the column height method is that time resolution

Copyright 1999 by the American Geophysical Union.

Paper number 1999JB900308.

0148-0227/99/1999JB900308\$09.00 is limited since column height is usually reported as an average value over several hours. Our method provides both the time history of mass emission rate and the orientation of the jetting using continuously measured seismic data. Such resolution allows us to quantify the sequence of events initiating an eruption.

We illustrate the new method by studying the cataclysmic eruption of Mount St. Helens. On the morning of May 18, 1980, a giant landslide exposed the cryptodome beneath the north flank of Mount St. Helens. The hot, pressurized magma exploded and over the next few minutes $3-4 \times 10^{11} \mathrm{~kg}$ of material surged northward over an area of approximately $600 \mathrm{~km}^{2}$. Although asymmetric explosive eruptions had been previously identified and termed "directed blasts" [LaCroix, 1930; Gorshkov, 1963], the devastation at Mount St. Helens brought the eruptive style to the attention of the volcanological community.

Many studies using a variety of data sets have addressed the Mount St. Helens blast, but none were able to include direct measurement of the mass emission rates. For the following practical and scientific reasons such a measurement would be useful:

1. Measurements of erupted mass quantify the hazard. Both the rate of eruption and the total mass of the products are useful measures of the size of an eruption as noted by Walker [1980]. Total erupted mass is a major criterion for the Volcanic Explosivity Index (VEI) [Newhall and Self, 1982] and such metrics consti- 
tute an important tool for formulating effective hazard management policies.

2. Various eruptive styles can be distinguished by their mass discharge rates. At Mount St. Helens the blast was unexpectedly and disproportionately devastating. This phenomenon was presumably due to the important role of momentum, or in the terminology of Walker [1980], the "violence" of this particular eruptive style. Measuring and documenting the mass emission rate of eruptions can clarify such features of eruptive styles.

3. Measurement of the erupted mass relates eruptive events to their products. The total mass in a unit can be estimated by mapping the deposits. Providing a history of the mass ejection over the course of the eruption could potentially associate the deposits with the processes that produced them. Seismic data can constrain the geometry of the source in addition to its time history.

4. Mass ejection rate $\dot{M}$ provides a direct constraint on dynamic models of an eruption. A number of fluid mechanical models of explosive eruptions have been developed [e.g., Wilson et al., 1980; Sparks et al., 1997] and the resulting flow solutions include predictions of mass flux. Accurate determination of $\dot{M}$ can test and calibrate these models.

The inversion for $\dot{M}$ begins with a series of equivalent forces that were previously found from the seismic data for May 18 [Kanamori and Given, 1982; Kanamori et al., 1984]. Seismic data have been analyzed to produce similar equivalent force systems during eruptions at other volcanoes [Uhira et al., 1994; Uhira and Takeo, 1994; Nishimura, 1995]. In this paper, we model the observed seismic pulses as thrusts due to the momentum flux of the erupted products. The momentum discharge rate is then converted to a mass discharge rate based on an independent estimate of the velocity. The resultant mass discharge rate is integrated over time to calculate the total mass corresponding to the observed seismic forces. We then reinterpret the eruption in light of this new data set. Our calculations allow us to evaluate the directionality of the blast.

The model used to connect the seismic observations with dynamical quantities is deliberately simplified to provide an analytical method of evaluating the data. As such, it inevitably omits features of a complex volcanic flow such as multiphase flow effects and fragmentation dynamics. Wherever possible, we justify the omissions through quantitative assessment. Such assessments are necessarily approximate and result in generous error estimates. Numerical treatment of the full solid and fluid dynamical systems could refine the results presented here and would be a logical extension of this work.

\section{Observations}

At 1532:11 UT (0832:11 LT) on May 18, 1980, seismic activity increased at Mount St. Helens and the cataclysmic eruption began. Within the next few min- utes three separate landslide blocks detached and dark plumes were photographed rising from the summit. About 40 seconds after the activity began, the largest slide block had reached the bottom of the mountain and a sudden expansion of light-colored material occurred. A light-colored, ground-hugging flow is first visible in photographs at this time [Hickson, 1990]. These rolling clouds are what is commonly referred to as the directed blast and have been variously interpreted as an underexpanded jet, pyroclastic flow or surge [Kieffer, 1981; Walker and McBroome, 1983; Waitt, 1984; Hoblitt and Miller, 1984]. The resulting deposit is called unit A and is subdivided into sedimentary layers A0-A3 [Waitt and Dzurisin, 1981; Hoblitt, 1989]. A giant mushroom cloud rose from the devastated area about $4 \mathrm{~min}$ after the seismic activity increased [Sparks et al., 1986]. The rapid ascent of the cloud was measured in satellite photos [Sparks et al., 1986] and reported by airline pilots [Rosenbaum and Waitt, 1981]. The fallout of this mushroom cloud formed layer A3, a fine, dark deposit distributed over the entire devastated area. A3 contains abundant shredded vegetation which suggests that the material flowed along the ground before being lofted into the mushroom cloud and eventually deposited [Sisson, 1995]. The next layer deposited (B1) was a "salt and pepper" combination of lithics and pumice [SarnaWojicki et al., 1981]. B1 marks the introduction of juvenile pumice into the deposits. It lies above $\mathrm{A3}$ almost everywhere and the boundary between the units is sharp [Waitt and Dzurisin, 1981]. This indicates that some time elapsed between the mushroom cloud and any pumiceous Plinian air fall. There was no Plinian column from the vent associated with the blast phase of the eruption [Criswell, 1987]. This important observation is reinforced by satellite images that show the blast column from the vent only reached a height of $\sim 6$ $\mathrm{km}$ above the mountain rather than expanding buoyantly into the upper atmosphere [Sparks et al., 1986]. Therefore the dynamics of the blast initiation can best be understood by studying the compressible, momentum driven flow in the gas-thrust region [Sparks et al., 1997] rather than by pursuing thermally driven plume theory.

These eruptive events were also observable seismically. Explosions and flows coupled to the ground to generate seismic waves. The various phases on the seismograms suggest distinct processes that can be modeled by systems of equivalent forces. Each process is distinguished by the frequency content and the geometry of the source (see Figure 1a.) Figure 2 reviews the evidence for a series of vertical single forces as first documented by Kanamori et al. [1984]. The near-field station at Longmire (Figure 2a) recorded the ground motion of the source directly and simple visual inspection suggests a source of the form illustrated in Figure $1 \mathrm{~b}$. The teleseismic data (Figure $2 \mathrm{~b}$ ) both characterizes the type of equivalent force system of the seismic source and quantifies the orientation and amplitude of those forces. 

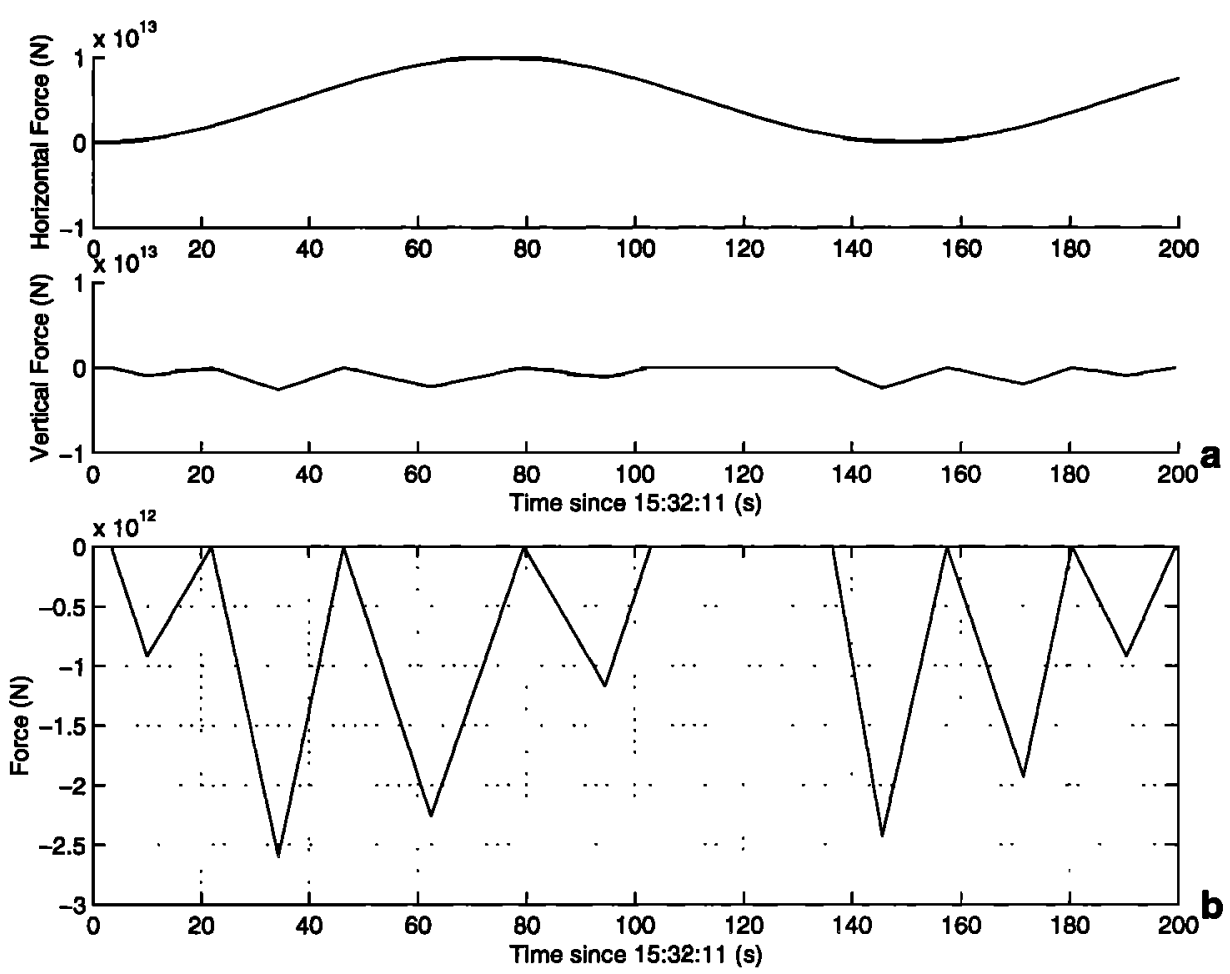

Figure 1. Time history of the seismic sources. (a) The seismic signal contains information about two distinct sources during the eruption. (Top) A horizontal long-period source inverted from the surface waves [Kanamori and Given, 1982] and (bottom) shorter period vertical pulses. (b) A magnified plot of the vertical pulses. These pulses are the sources of the seismic waves in Figure 2 [Kanamori et al., 1984].

The key observation for determining the force system is the azimuthal invariance of the $P$ waves (Figure 2b). The nearly identical amplitudes and waveforms at all azimuths strongly suggest vertical single force sources. This pattern of radiated energy is incompatible with the four-lobed pattern of the double couple fource model used for most tectonic earthquakes. Furthermore, the large amplitudes of the $P$ waves relative to the $S$ waves indicate that the inclination of the source must be at least $60^{\circ}$ from the horizontal. This estimate is further refined by observing that the dominant period of the $S$ waves in Figure 2 is too long to be generated by the $P$ wave source. If the $P$ wave source generated any $S$ waves, they are obscured by the longer period signal, that is, they are smaller amplitude than the traces in Figure 2b. Therefore the inclination of the source must be much greater than $60^{\circ}$ [Kanamori et al., 1984]. We emphasize that the vertical orientation of the force is constrained by the azimuthal symmetry of the radiation patttern, rather than merely by the fact that the waves are observed on the vertical component.

The magnitudes of the single force sources are estimated using the amplitudes of the far-field $P$ waves. The resulting source function in Figure $1 \mathrm{~b}$ is two distinct sequences of 20 to $30 \mathrm{~s}$ pulses with vertical component amplitudes of the order of $10^{12} \mathrm{~N}$. The geometric constraints require that any horizontal components of these pulses have amplitudes less than half the vertical values. The largest pulse of the first sequence peaks at $\sim 1532: 45$ which is the time that a sudden increase in the ash cloud height occurs in the photos of the eruption. Further support for this source inversion is provided by more detailed analysis of the seismic data including a full consideration of the radiation pattern [Kanamori et al., 1984; Burger and Langston, 1985]. Similar lines of reasoning for the surface wave data yield a separate long-period (300 s) horizontal source that has been associated with traction at the base of the landslide [Kanamori and Given, 1982]. No direct measure of the horizontal component of the 20 to $30 \mathrm{~s}$ pulses is possible because the larger, longer period landslide dominated the transverse component signal.

Estimates of the erupted mass from both the geological and seismological observations are used in this paper to link the two types of data. The mass discharged at each stage of the eruption can be estimated by calculating the total mass of the corresponding geological deposits while assuming that the mass of the associated volatiles is negligible. Although gas was volumetrically and dynamically important, it is unlikely that its mass exceeded a few weight percent when the mixture was originally erupted from the vent [Eichel- 

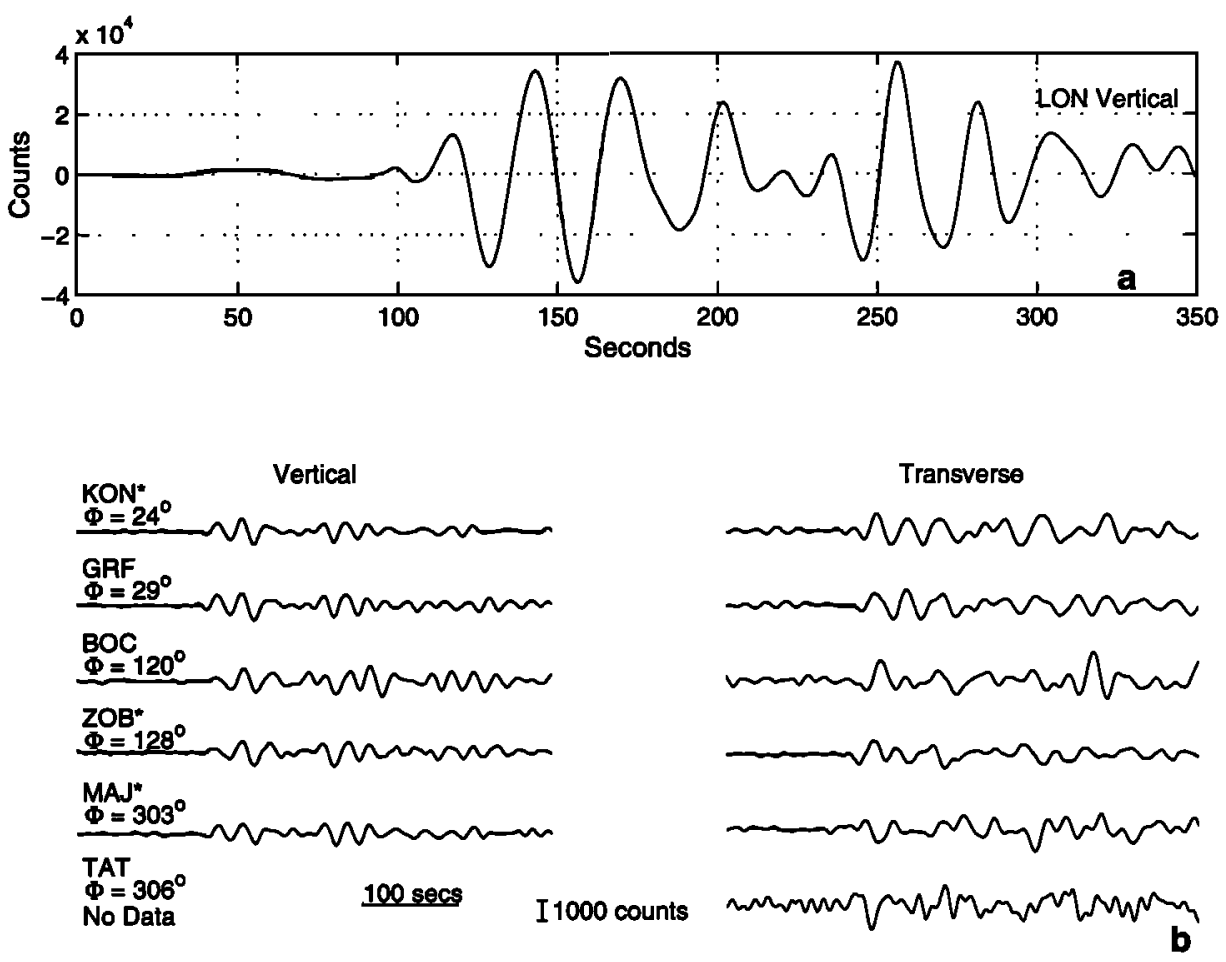

Figure 2. Body wave data for May 18, 1980 initial eruptive events. (a) Nearfield vertical record from the WWSSN station at Longmire (LON). The distance $\Delta$ from the source was $67 \mathrm{~km}$ and the azimuth $\Phi$ was $26^{\circ}$. (b) Far-field recordings of the $P$ and $S$ arrivals at SRO and ASRO stations arranged according to azimuths, $\Phi$. $P$ and $S$ waves are observable on the vertical and transverse components, respectively, and the traces are aligned on the Jeffreys-Bullen arrival times of an event beginning at 1532:11. All amplitudes are normalized for geometric spreading to the distance of GRF, $\Delta=76.2^{\circ}$. The instrument at TAT had the polarization of the horizontal components reversed in May 1980, therefore the observed trace is inverted in this figure. ASRO stations are denoted by asterisks. Their amplitudes are reduced by 2 and the traces are shifted $4 \mathrm{~s}$ to the left for comparison with the SRO stations. At a period of $25 \mathrm{~s}$, an amplitude of 1000 counts in this figure corresponds to $0.2 \mu \mathrm{m}$ of ground motion.

berger and Hayes, 1982; Kieffer, 1981]. The total blast deposit (unit A), which includes both country rock and juvenile dacite, had a volume of about $0.19 \mathrm{~km}^{3}$ [Moore and Sisson, 1981]. This value is probably an overestimate of the material erupted from the vent since significant erosion and entrainment may have occurred as the blast cloud traveled down the slope of the mountain [Kieffer and Sturtevant, 1988]. The average density has been estimated as between 1660 [Hoblitt et al., 1981] and $2180 \mathrm{~kg} / \mathrm{m}^{3}$ [Glicken, 1996]. The mass of the blast is therefore estimated to be $3.2 \times 10^{11}-4.1 \times 10^{11} \mathrm{~kg}$. This value can be put into perspective by noting that the total mass of the juvenile magmatic products erupted on May 18, 1980 at Mount St. Helens is $5.2-7.0 \times 10^{11}$ kg [Christiansen and Peterson, 1981; Friedman et al., 1981].

\section{Force Balance}

In order to understand the forces generating the seismic waves, we consider the balance of forces on the magma. The relevant dynamics are analyzed by modeling the magma as a fluid that is accelerated out of the ground by the depressurization that accompanied the landslide. The landslide removed the lid from a body of fluid under high pressure, and therefore the fluid was rapidly ejected.

We define a control volume around the magma in the ground (Figure 3). The geometry is simplified by representing the magma body as a trapezoidal volume. Each surface represents the area on which the resolved forces in the indicated direction act, rather than the literal wall of the magma body. The sum of the forces on the fluid equals the rate of change in the momentum of the fluid according to Newton's second law. The stresses acting on the fluid are gravitational and the pressures from the interactions with the atmosphere and the solid earth. The momentum can change in the fluid volume $V$ either due to changes in the velocities internally or mass (and hence momentum) exiting through the surfaces of the volume. The sum of the forces exerted on the fluid $\sum F_{f l}$ is 
II

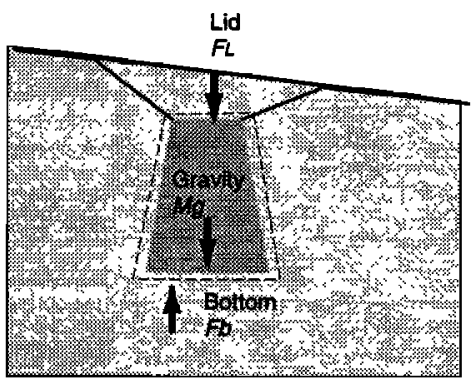

III

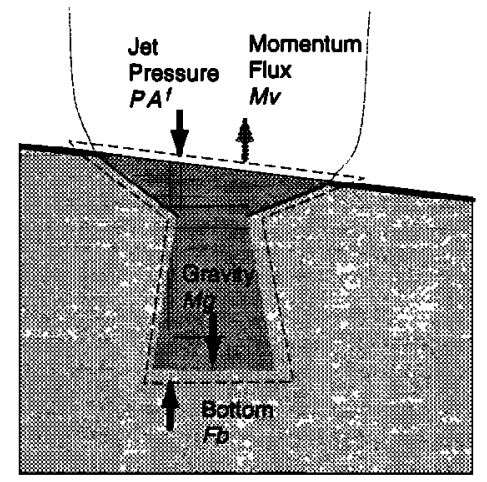

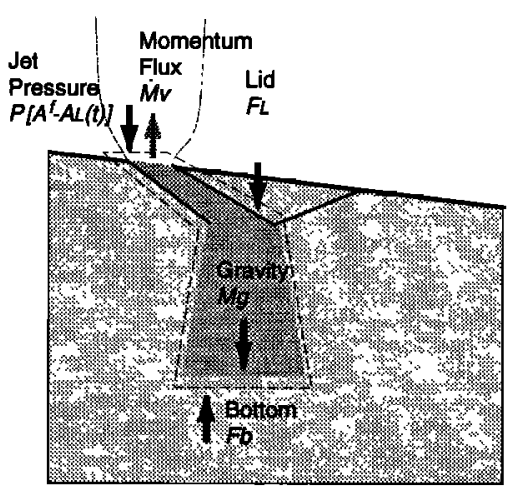

IV

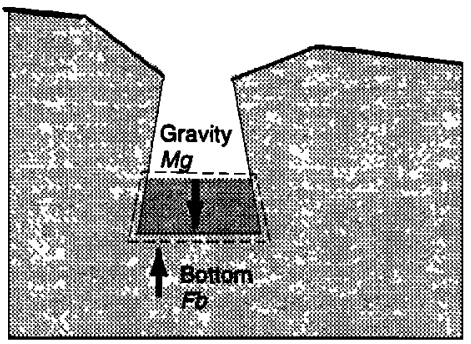

Figure 3. Cartoon of a control volume (dashed line) around a magma body (dark region) and the forces between the solid earth (lightly shaded area) and the magma acting during a single eruptive pulse. (I) The initial conditions. (II) The lid is removed in the landslide. (III) The fully developed jet. (IV) The final conditions.

$\sum F_{f l}=-M g+\sum F_{a t}+\sum F_{s e}=\frac{d}{d t} \int_{V} \rho u d V-\dot{M}_{f l} v$

where $M$ is the mass of the fluid, $g$ is the magnitude of the gravitational acceleration, and the forces acting on the surface of the control volume are $F_{a t}$ from the atmosphere (including the part of the jet which is above the ground) and $F_{s e}$ from the solid earth. The velocity of the fluid exiting the control volume is $v, u$ represents the velocity inside the control volume and $\rho$ is the density of the fluid. The rate at which the mass of the fluid inside the control volume changes is $\dot{M}_{f l}$. Note that $\dot{M}_{f l}=-\dot{M}$ as the mass emission rate is positive when the mass in the control volume is decreasing. Forces are positive upwards.

Transient forces from the eruption that are coupled to the solid earth generate seismic waves. It is by observing these waves that we are able to determine the source parameters of the eruption so the "seismically observed force" $F$ will be defined as

$$
F \equiv-\sum F_{s e}
$$

In this paper, we are interested in analyzing the observed vertical short-period seismic forces since the hor- izontal ones are not observed for the Mount St. Helens blast. Therefore we will only consider the vertical force balance. Note that the only explicitly time dependent term in (1) is the time derivative of the momentum inside the control volume. We neglect this change of internal momentum, to derive a quasi-static approximation to $(1)$,

$$
\sum F_{f l}=\dot{M} v \text {. }
$$

A full discussion of the validity of the quasi-static approximation for eruptive events is postponed until section 6.

Equation (3) is often referred to as the rocket equation [Thompson, 1972] since the sum of forces propelling a rocket upwards is equal to the momentum discharge rate of the fuel behind it. The blast at Mount St. Helens can be viewed as an inverted rocket where the momentum of the mass thrust into the air balances a net downward force into the ground.

The atmospheric force term $\sum F_{a t}$ accounts for the fact that a supersonic jet can be "underexpanded", that is, at a pressure significantly above atmospheric at the vent. The net force of the jet on the fluid in the chamber is then the pressure in the jet $P$ multiplied by the area of the vent. The sum of the atmospheric forces during the eruption is therefore 


$$
\sum F_{a t}=-P A
$$

where $A$ is the area of the vent. $F$ can now be derived in terms of dynamic quantities by combining (1), (2), (3), and (4),

$$
F=-(M g+\dot{M} v+P A) .
$$

Equation (5) is a quasi-steady description of the force balance. It relates the observed force to the instantaneous dynamics of the jet but gives no insight into how this force varies over time. In order to understand the shape of the observed seismic pulses it is useful to reconsider the forces in terms of the geometry during depressurization. An eruptive pulse is divided into four representative stages in Figure 3.

Before the eruption (stage I) the magma is pressurized and at rest. We define the magnitude of the force exerted by the country rock at the top of the magma body as $F_{L}$. This is the force exerted by the lid prior to eruption and includes both the strength and weight of the caprock. The magnitude of the force on the bottom of the magma is denoted by $F_{b} . F_{b}$ and $F_{L}$ are defined to be the absolute values of the forces, and we explicitly note their signs in the following equations. For the purposes of the qualitative discussion of the time history of the force in this section, the vertical forces on the sides of the control volume are neglected. The difference between $F_{L}$ and $F_{b}$ is the net vertical force of the magma on the solid earth, i.e., the seismic force $F$.

$$
F=F_{L}-F_{b} \text {. }
$$

During stage II the lid is removed in the landslide, the restraining force $F_{L}$ decreases in time, and a jet forms. When the lid is removed, the pressure at the bottom of the magma body does not begin to fall until after a time $\tau$ required for the pressure drop, or rarefaction wave, to travel to the bottom of the magma body. During this time the lid continues to be accelerated. By the time the rarefaction wave reaches the bottom of the chamber, the pressure is dropping even faster at the top. Therefore the downward force increases in magnitude throughout the lid removal process (stage II). Stage III in Figure 3 is when the lid is completely removed and the jet is fully developed. As the pressure in the chamber is released, the jet wanes and the magnitude of $F$ decreases. The final phase, stage $I V$, occurs after the eruption. Only the weight of the remaining magma exerts a force on the ground. During the next pulse the process is repeated. We envision the initiation of the Mount St. Helens eruption to be a series of such depressurization events as the cap to the cryptodome broke into pieces. Each pulse released a section of the cryptodome that was separated from the other sections either by country rock or unvesiculated zones. We speculated that these separate regions may correspond to the heterogeneously vesiculated zones described by Hoblitt and Harmons [1993].
The geometric and dynamic considerations can be summarized by the following four equations:

$$
\begin{array}{lrrrr}
\text { Stage I } & F= & F_{L}^{0}-F_{b}^{0} & = & -M_{0} g \quad(7 \mathrm{a}) \\
\text { Stage II } & F= & F_{L}(t)-F_{b}(t)= & -\{\dot{M} v+ \\
& & & P\left[A^{f}-A_{L}(t)\right] \\
& & & +M(t) g\} \quad(7 \mathrm{~b}) \\
& & & \\
\text { Stage III } & F= & -F_{b}(t) & -\left[\dot{M} v+P A^{f}\right. \\
& & & +M(t) g] \quad(7 \mathrm{c}) \\
& & & & \\
\text { Stage IV } & F= & -F_{b}^{f} & -M_{f} g \quad(7 \mathrm{~d})
\end{array}
$$

where $F_{L}^{0}, F_{b}^{0}$, and $M_{0}$ are the initial lid force, bottom force, and mass, respectively, and $F_{b}^{f}$ and $M_{f}$ are the final bottom force and mass of the magma. $A^{f}$ is the final area of the vent and $A_{L}$ is the area that is covered by the lid. The force history of the entire process is shown schematically in Figure 4.

According to the above theory, the seismic force should be a function of the weight of the magma erupted, the thrust of the blast and the pressure in the jet. However, the seismological measurements are bandwidth limited. For the long, complex event on May 18, 1980, the gravitational unloading occurred over the entire 100 second series of pulses. In the far-field, such a long-period signal was obscured by the instrumental and ground noise. In the near-field (LON), the short record available shows spurious oscillations when the instrument is deconvolved [Kanamori et al., 1984] and these artifacts obscure any long-period signal. Therefore we neglected the gravitational terms $M g$ in (7a)-(7d) when analyzing the instrumentally recorded data for this event even though the magnitude of the gravitational unloading at the source may be as large as the other terms. For the same reason, the small vertical component of the longer period landslide force is neglected in the momentum balance. Fortunately, the photographic record documents that pulsations in the thrust and jet pressure occurred at somewhat shorter periods $(\sim 20-30 \mathrm{~s})$ [Nielsen et al., 1989; S. Malone, Mount St. Helens timing, unpublished data, 1998]. These shorter period waves were recorded by the seismometers.

We have now related the observed seismic force $F$ to the momentum term $\dot{M} v$ and the jet force $P A$ at each stage of the eruption and obtained a qualitative sense of the time variation of these variables. The only assumption about the fluid dynamics is that internal momentum changes are negligible. In order to proceed further we need to relate the jet force $P A$ to the momentum discharge rate $\dot{M} v$ which requires a specific model of the flow.

\section{Flow Model}

We model the flow as an ideal gas expanding isentropically with a specified mass fraction $\phi$ of suspended particles. We follow Kieffer [1981] in modeling the fully developed flow geometry as a nozzle in which the 


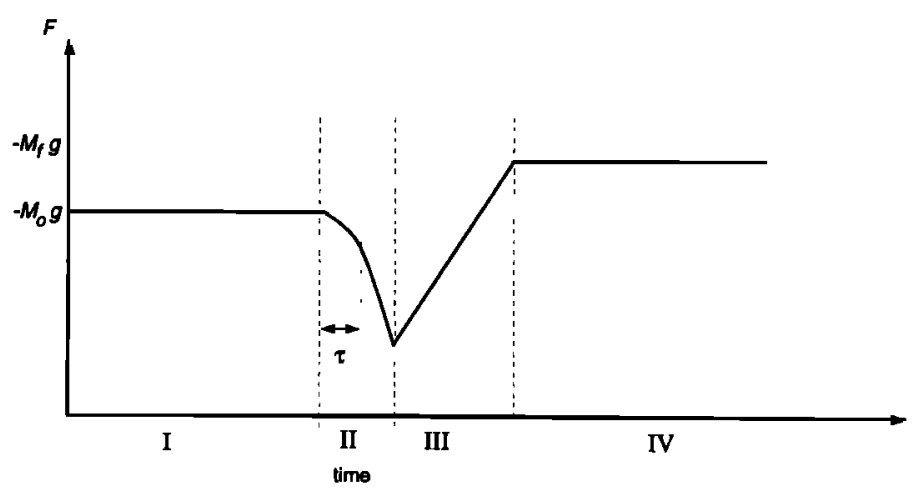

Figure 4. Schematic of seismic force $F$ corresponding to the stages of Figure 3 . The initial force is the preeruptive weight of the magma. Since a seismograph only records changes in $F$, this
baseline value is arbitrary.

discharge is a supersonic jet. For flows like the blast of Mount St. Helens that are volumetrically primarily gas, the equation of state of the mixture can be derived by describing the fluid as a mixture of two gases, one of which (the solid phase) is incompressible. Such a fluid is known as a dusty gas, or pseudogas, and is used as a model fluid in engineering applications ranging from solid fuel rocket engines to pipeline technology [Rudinger, 1980]. This formulation allows us to invoke a number of standard results from fluid mechanics. In particular, we can relate the flow parameters at the exit to the local sound speed. The ratio of the pressure term to the momentum discharge rate is then simply a function of the Mach number.

The equation of state of an equilibrium solid-gas mixture with noninteracting particles is [Rudinger, 1980]

$$
P=\rho R_{M} T /(1-\xi)
$$

where $R_{M}$ is an ideal gas constant for the mixture, $\rho$, $T$ and $P$ are the density, temperature and pressure in the mixture, respectively, and $\xi$ is the volume fraction of solids. The term "equilibrium" in this context means that the solid and gas phases flow at the same velocity (see Appendix A).

The solid volume fraction is related to the mass fraction $\phi$ by

$$
\xi=\phi \frac{\rho}{\rho_{s}},
$$

where $\rho_{s}$ is the density of the solid. This equation of state is an ideal gas modified to account for the volume of an incompressible solid phase. In the limit of $\xi \rightarrow 0$ the fluid becomes an ideal gas as modeled by Kieffer [1981]. The isentropic sound speed $c$ corresponding to (8) is [Rudinger, 1980]

$$
c^{2} \equiv\left(\frac{\partial P}{\partial \rho}\right)_{s}=\frac{\Gamma R_{M} T}{(1-\xi)^{2}}=\frac{\Gamma P}{\rho(1-\xi)},
$$

where $\Gamma$ is defined to be the ratio of the specific heats for the mixture and is analogous to the adiabatic constant for an ideal gas. The specific heat of the mixture is the weighted average of the specific heats of the gas at constant pressure $c_{p}$ and volume $c_{v}$ and the specific heat of the solid $c_{s}$.

$$
\Gamma=\frac{(1-\phi) c_{p}+\phi c_{s}}{(1-\phi) c_{v}+\phi c_{s}}
$$

The solid mass fraction $\phi$ is over $90 \%$. The maximum whole rock $\mathrm{H}_{2} \mathrm{O}$ content proposed for the preeruptive magma is 3-5 wt \% based on both petrological and isotopic evidence [Hoblitt and Harmons, 1993]. A fraction of the $\mathrm{H}_{2} \mathrm{O}$ remained trapped in vesicles and therefore dynamically inactive. Therefore the minimum value of $\phi$ is $95 \%$. The solid mass fraction $\phi$ remains constant throughout the flow of an equilibrium dusty gas even though the solid volume fraction $\xi$ decreases significantly. These high values of $\phi$ cause the adiabatic constant $\Gamma$ to be nearly 1 . In the limit of $\Gamma \rightarrow 1$, the nondissipative (isentropic) expansion of the fluid through a nozzle is isothermal. Rewriting (10),

$$
P A=(1-\xi) \rho c^{2} A \quad ; \quad \Gamma=1 .
$$

The momentum discharge rate at the exit, that is, the flux through the top surface of the control volume multiplied by the area, is

$$
\dot{M} v=\rho v^{2} A .
$$

Therefore the ratio of the pressure term to the momentum discharge rate is

$$
\frac{P A}{\dot{M} v}=\frac{(1-\xi) c^{2}}{v^{2}}=\frac{(1-\xi)}{\mathcal{M}^{2}}
$$

where $\mathcal{M}$ is the exit Mach number defined as the exit velocity $v$ divided by the local sound speed $c$. Since $1 \geq \xi \geq 0$,

$$
0 \leq P A \leq \dot{M} v \frac{1}{\mathcal{M}^{2}} .
$$

Until now we have treated only the mass discharge 
rate of the bulk fluid. When we compare our results with the geological deposits, we will want to limit ourselves to the mass discharge rate of the solid phase. According to the definition of mass fraction $\phi$, the solid mass discharge rate is simply $\phi \dot{M}$. As already noted, the mass fraction is nearly unity for the blast, and approximating the solid mass discharge rate as the total mass discharge rate is reasonable.

Equation (15) relates the pressure at the top of the control volume to the mass discharge rate. We combine $(7 \mathrm{~b}),(7 \mathrm{c})$, and (15) with gravity neglected as discussed above and incorporate the definition of Mach number $\mathcal{M} \equiv v / c$, to obtain the inequality

$$
\dot{M} c \mathcal{M} \leq-F \leq \dot{M} c \mathcal{M}+\frac{\dot{M} c}{\mathcal{M}} .
$$

Since the seismically observed quantity is the force $F$, the inequality can be more usefully written as

$$
\frac{-F}{c \mathcal{M}} \geq \dot{M} \geq \frac{-F}{c \mathcal{M}\left(1+1 / \mathcal{M}^{2}\right)}
$$

If we knew the exit Mach number $\mathcal{M}$ and sound velocity $c$ as a function of time as well as the force history exactly, we could estimate the mass discharge rate within a factor of $\left(1+1 / \mathcal{M}^{2}\right)$. Unfortunately, it is not possible to know the precise values of these parameters for real eruptions, but we can make reasonable estimates for well-documented cases like Mount St. Helens using the considerations below.

\section{Estimation of Parameters}

The force $F$ as a function of time is approximately known from seismic data as detailed in section 2 . We must estimate the Mach number $\mathcal{M}$ and the sound velocity $c$ in order to utilize the bounds imposed by (17).

The Mach number $\mathcal{M}$ at the top of the control volume is computed by estimating the expansion in the crater (see Appendix B). The area of the supersonic flow was probably smallest at the bottom of the crater. This point of minimum cross-sectional area is termed the "throat" in gas dynamics, and quantities measured there are denoted by a star subscript. The ratio of the area at the top of the crater to the area of the vent at the bottom of the crater walls as measured on a geological map [Lipman, 1981] is a rough measure of the expansion. The crater geometry during the blast was certainly different than the posteruptive topography since the mountain was undoubtedly changed during the 9hour long violent eruption, so we consider a wide range of possible expansion ratios $A / A_{*}$. We estimate that the area of the jet increases by a factor between 4 and 40 which corresponds to Mach numbers of 2.1-3.4 assuming that the solid volume fraction at the throat $\xi_{*}$ is between 0 and 0.5 (See Appendix B.) Since these values are based on approximate estimates, we will round the numbers and consider a range of $\mathcal{M}$ from 2 to 3.5 in this work. Note that this estimate of $\mathcal{M}$ is for the fully open vent of stage III. During stage II the jet expands less and the exit Mach number is smaller. Using values of $\mathcal{M}$ from 2 to 3.5 for both stages therefore results in a conservative estimate of the mass discharged.

The sound velocity $c$ is estimated by rewriting (10) in terms of temperature [Marble, 1970; Rudinger, 1980]

$$
c=\sqrt{\frac{\Gamma(1-\phi) R T}{(1-\xi)^{2}}},
$$

where $R$ is the ideal gas constant for the volatile. We assume the gas phase was primarily $\mathrm{H}_{2} \mathrm{O}$ with $R=461.5$ $\mathrm{J} / \mathrm{kg} / \mathrm{K}$. As can be seen from (18), the sound velocity is weakly dependent on the temperature and the volatile content of the erupting column. Kieffer [1981] proposed a cool reservoir of $600 \mathrm{~K}, 3.9 \mathrm{wt} \% \mathrm{H}_{2} \mathrm{O}$ and a density at the throat corresponding to a solid volume fraction $\xi_{*}$ of about $30 \%$. The expansion ratios $A / A_{*}$ considered above reduce $\xi$ to at most $4 \%$ at the exit and the corresponding sound velocity is $\sim 110 \mathrm{~m} / \mathrm{s}$. If Kieffer's model is adjusted to magmatic temperatures, that is, $T=1200 \mathrm{~K}$, the sound velocity is $\sim 150 \mathrm{~m} / \mathrm{s}$. Eichelberger and Hayes [1982] prefer a hotter, drier mixture of $1173 \mathrm{~K}, 0.7$ to $1.7 \mathrm{wt} \% \mathrm{H}_{2} \mathrm{O}$ and at least 67 vol \% solid throughout the flow. The volume fraction estimate of Eichelberger and Hayes [1982] is the fraction of $\mathrm{H}_{2} \mathrm{O}$ trapped in the vesicles of pumice, rather than the dynamically active gas outside the clasts and therefore is an inappropriate value for the fluid fraction of the dusty gas. Individual pumice fragments were entrained in a gas-rich flow that is being modeled here. We consider a range of possible values of $c$ from 100 to $150 \mathrm{~m} / \mathrm{s}$.

The values of $\mathcal{M}$ and $c$ considered here correspond to exit velocities of $200-525 \mathrm{~m} / \mathrm{s}$. The exit velocity of the fluid at the top of the crater is poorly constrained by observations. The position of the front of the lateral blast has been analyzed in photos taken by G. Rosenquist [Voight, 1981]. Depending on the choice of timing schemes of the photos, the lateral blast front is estimated to have moved northward at $\sim 50-100 \mathrm{~m} / \mathrm{s}$ within $1 \mathrm{~km}$ of the vent. However, the horizontal velocity of the blast downhill of the vent provides no constraint on the vertical velocity at the vent. Even if the blast were ejected in the direction of final motion, Kieffer and Sturtevant [1984] showed that front velocity is smaller than the fluid velocity at the exit.

The parameters above allow us to invert the force $F$ for $\dot{M}$. For $F$ in units of Newtons and $\dot{M}$ in kilogram per second, the numerical equivalent to (17) is

$$
\frac{-F}{200} \geq \dot{M} \geq \frac{-F}{570} .
$$

Figure 5 shows the total mass erupted computed from (19) using the observed forces and integrating the resulting $\dot{M}$ over time. The mass of the blast deposit as estimated from the geological evidence is also plotted 

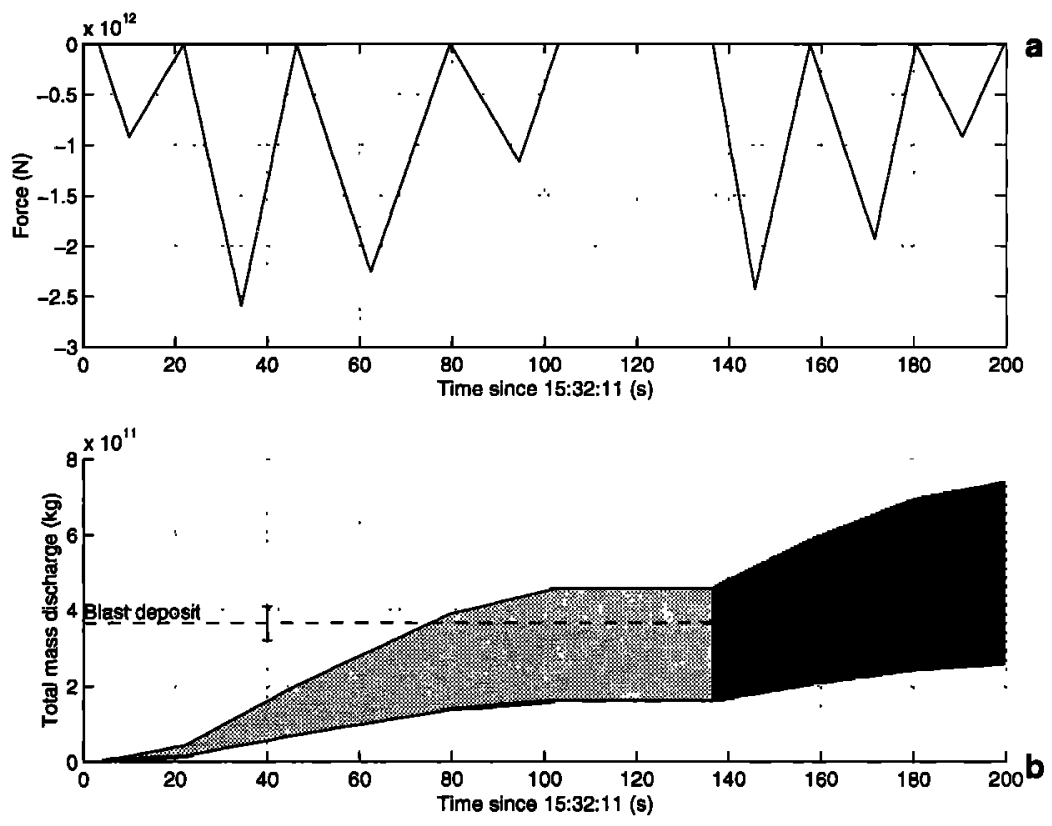

Figure 5. (a) The forces inverted from the seismic data of Kanamori et al. [1984]. (b) Estimate of mass erupted using data in Figure $5 \mathrm{a}$ and(17). The upper bound of the shaded region corresponds to $c=100 \mathrm{~m} / \mathrm{s}, \mathcal{M}=2$ and the upper bound of (17); the lower bound corresponds to $c=150 \mathrm{~m} / \mathrm{s}$, $\mathcal{M}=3.5$ and the lower bound of (17). The mass calculated from the second set of pulses is shaded darker because these later explosive events are not conclusively related to the blast (see text).

as a dashed line for comparison. Around 1.6-4.6 $\times 10^{11}$ $\mathrm{kg}$ of material was discharged vertically over the first $100 \mathrm{~s}$. An additional $1-2.8 \times 10^{11} \mathrm{~kg}$ corresponds to the second set of forces.

\section{Discussion}

At this point in our study it is appropriate to ask how well-constrained is the force-time history by the seismic data. What errors are introduced in determining this function? Limited bandwidth seismic data does not tightly constrain the magnitude of the equivalent forces. However, the integrated force over time, or impulse, is robustly determined. Since the tctal mass erupted is a function of the impulse, the errors from the seismic data are negligible in comparison to the errors in estimating the parameters $\mathcal{M}$ and $c$. We can verify this claim by repeating the calculation of Figure 5 using an alternative inversion for the force-time history. The force-time function in Figure 6a is inverted from the far-field waveforms rather than the near-field data that was used for Figures 1b and 5a [Kanamori et al., 1984]. Figure 6 shows that the impulse for the complete event, and hence the total erupted mass, is the same for these two models.

We can also quantify the error implicit in the quasistatic approximation. The internal momentum term acts as a storage term in the equations. When the boundaries are changed, for example, the lid is removed, the internal momentum of the control volume is increased. The pressure drop travels through the body as a rarefaction wave. When it reaches the opposite boundary, momentum is transferred from inside the control volume to the outside by exerting a force on the bottom boundary. We can quantify the effect of internal momentum changes on the calculated total mass $M$ by integrating the force balance equation over time from the beginning of the event $t_{0}$ to the end of the event $t_{f}$.

$$
\int_{t_{0}}^{t_{f}} P A d t-\int_{t_{0}}^{t_{f}} F d t=\left.\int_{V} \rho u d V\right|_{t_{0}} ^{t_{f}}+M \bar{v}
$$

where $\bar{v}$ is an average exit velocity defined by

$$
\bar{v} \equiv \frac{\int \dot{M} v d t}{M} .
$$

If we assume that the internal momentum before (stage I) and after the event (stage IV) are both negligible, then the net effect of the internal momentum term is negligible. The total mass erupted $M$ is robustly determined by neglecting internal momentum. There may be some error in the $\dot{M}$ computed in the intermediate stages, but the errors cancel in the final integration.

The initial hypothesis that the forces can be modeled as a series of jets must also be reexamined before the calculated results can be interpreted. How applicable is the cartoon of Figure 3 to each of the observed pulses? Some alternative processes are considered below.

The landslide undoubtedly was not a strictly horizontal phenomenon. How do we know that the pulses we observe are not simply responses to irregularities in the landslide movement? The landslide radiated seismic en- 

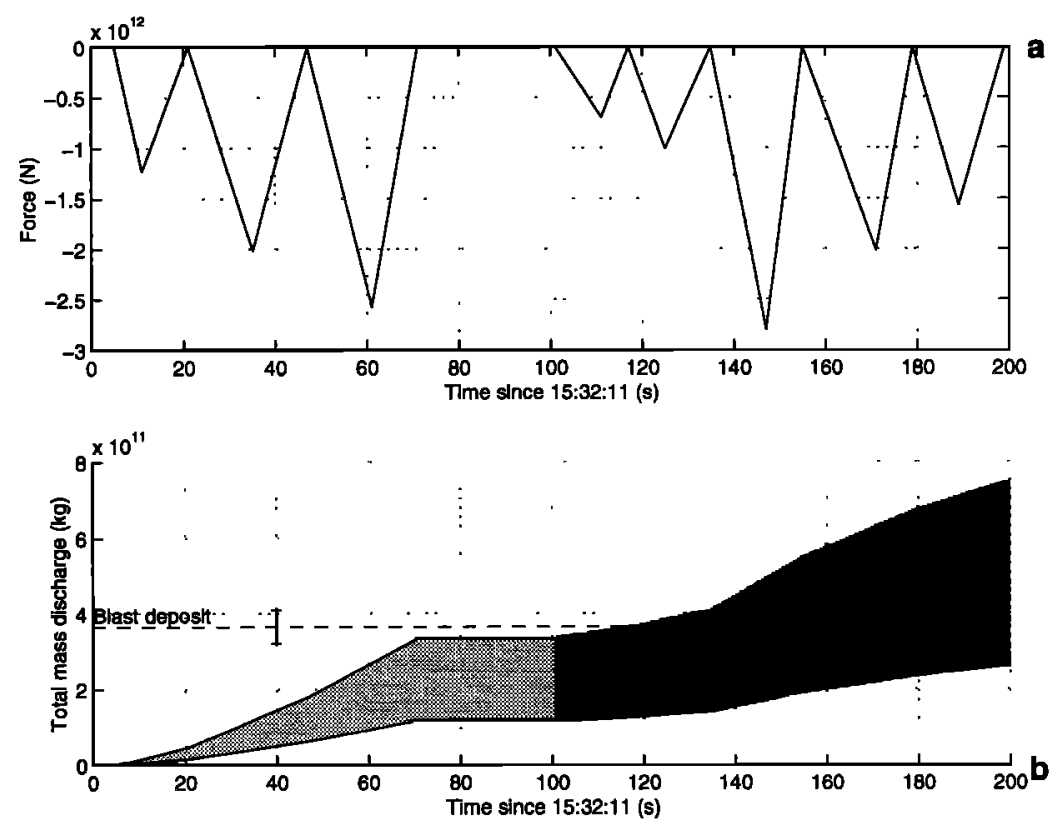

Figure 6. (a) An alternative force model from the seismic data of Kanamori and Given [1982]. (b) Estimate of mass erupted using data in Figure 6a and (17). Bounds are the same as in Figure 5. Note that the total mass at $200 \mathrm{~s}$ calculated here is the same as in Figure 5 since the total impulse is well constrained by the seismic data.

ergy by shearing the ground at its base. The equivalent forces on the elastic medium were single forces parallel to the ground. Even if the landslide moved over steep topography in places, an equivalent force at an angle $>60^{\circ}$ to the horizontal is highly unlikely to be caused by the landslide at any time.

Another objection that might be raised is that the decreasing train from 22 to 103 seconds might be reflections due to only one initial explosive event. However, a simple seismic reflection from the Moho or some other geological feature would have occurred significantly faster than the observed 25-30 s between peaks. An atmospheric reflection would have been slower than the observed period by at least a factor of three. Another possibility is that the pulses are reverberating depressurization (rarefaction) waves in the magma body. It has been proposed that the main magma body was at approximately $7-9 \mathrm{~km}$ below the cryptodome [Scandone and Malone, 1985]. If the waves traveled at the sound velocity through the unfragmented magmatic conduit connecting the upper and lower chambers, the two-way travel time might be the requisite $25-30$ secs. However, these reflections would result in further rarefaction waves and therefore a continually decreasing force rather than the observed series of triangular pulses. The increasing stage of the downward force (stage II) requires the removal of a lid. Each pulse must be a separate explosive event with attendant lid removal.

The coincidence of the first set of thrusts over the first 2 min with the photographically documented expansion suggests that the thrusts arose from the depres- surization of sections of the cryptodome. It has been suggested that the second set of pulses from 1534.6 to 1535.3 UT is the result of a distinctly different process. Moore and Rice [1984] observed that at approximately the time of these forces a plume rose from the Spirit Lake or Toutle River area 8-10 km north of the vent. The most sustained and intense infrared radiation was also observed during 1534.4-1534.7 [Moore and Rice, 1984]. Moore and Rice [1984] interpret these events as either the unroofing of previously undisturbed cryptodome material in the landslide blocks or explosions produced by the interaction of the hot material with the water of the Toutle River drainage. Either interpretation of Moore and Rice [1984] would imply that the momentum balance would be somewhat different than in the model presented in the Momentum Balance section and the appropriate velocities would be unconstrained. However, the secondary explosion hypothesis of Moore and Rice [1984] is controversial. Hoblitt [1989] proposes that the late explosions accompanied the removal of slide block III, the former top of Mount St. Helens. In this scenario, our original analysis remains applicable. Secondary unroofing event occurred 2 min after the start of the eruption and produced jets that were obscured by the expanded blast cloud. Recognizing the ambiguity of the field evidence, we include the mass from the secondary explosions in darker shading in Figure 5b but acknowledge that the relationship between this mass and the deposits is uncertain.

The total mass of the eruption on May 18 was $7-$ $9 \times 10^{11} \mathrm{~kg}$ (see Table 1.) The calculations of this 
Table 1. Mass Comparison

\begin{tabular}{lccc}
\hline & $\begin{array}{c}\text { Mass } \\
10^{11} \mathrm{~kg}\end{array}$ & $\begin{array}{c}\text { Mass } \\
\text { \% Blast Deposit }\end{array}$ & $\begin{array}{c}\text { Mass } \\
\text { \% Total Erupted }\end{array}$ \\
\hline Blast deposit & $3.2-4.1$ & 100 & $35-60$ \\
Total juvenile & $5.2-7.0$ & $125-220$ & $60-100$ \\
Total erupted & $7-9$ & $170-280$ & 100 \\
Computed mass a & $1.6-4.6$ & $40-90$ & $20-65$ \\
Computed mass b & $2.6-7.4$ & $65-230$ & $30-105$ \\
\hline
\end{tabular}

A comparison of the mass calculated for various stages of the eruption by various methods. Blast deposit mass is the mass of Unit $A$ as discussed in the text. Total juvenile is the sum of the masses of magmatic products from all phases of the May 18 eruption [Christiansen and Peterson, 1981; Friedman et al., 1981]. Total erupted is the sum of masses from all phases of the eruption, including the non-juvenile component of the blast. Computed mass $a$ and $b$ are the seismologically determined, vertically ejected mass. Computed mass a corresponds to the first 120 seconds of data; computed mass $b$ includes both sets of pulses in Figure 5.

study imply that between 1.6 and $4.6 \times 10^{11} \mathrm{~kg}$, that is, $20 \%-65 \%$ of the total mass of the eruption, was discharged vertically at a rate of $\sim 2-6 \times 10^{9} \mathrm{~kg} / \mathrm{s}$ within the first $2 \mathrm{~min}$ of the eruption. A second set of explosions is equivalent to an additional $1-2.8 \times 10^{11} \mathrm{~kg}$ of material. Including the second set of explosions brings the vertically erupted mass total to $2.6-7.4 \times 10^{11} \mathrm{~kg}$.

\section{Interpretation}

The term "lateral blast," which is usually used to refer to the initial explosive event of the Mount St. Helens eruption, implies a predominantly horizontal flow. We can use the mass calculation above to evaluate this implicit assumption of the blast's direction. Figure 5 indicates that at least $30 \%$, or at least $65 \%$ if the second set of events is included, of the mass of the blast deposit was involved in the vertical thrust events. We acknowledge that there may be substantial errors in both the geological deposit measurements and the seismic model, but even if we allow for this uncertainty, a substantial fraction of the blast deposit mass was jetted vertically from the vent. We reemphasize that this result is independent of the assumed crater geometry. As discussed in section 2, the seismic data constrains the measured component of the force, and hence the measured mass, to be subvertical. If each of the vertical pulses represents a thrust, the mass balance considerations make it unreasonable to assume that all of the blast deposit is composed of material that was initially ejected horizontally. Much, if not most, of the blast was initially ejected "vertically," that is, at an angle much greater than $60^{\circ}$ from the horizontal. The destructive "lateral" blast was either composed of only a fraction of the mass in the deposit or the vertical blasts were redirected by the geometry of the crater and the force of gravity. Either possibility has implications for the quantitative understanding of directed blasts and their deposits.

\section{Conclusions}

This case study of Mount St. Helens has shown that the thrust inversion technique is an effective tool for measuring mass discharge rate. The mass ejection history can be computed from the seismic data using only two parameters: Mach number and sound velocity. We now return to the original motivations for measuring $\dot{M}$ mentioned in the introduction in order to evaluate the importance of our results for the case of Mount St. Helens. We have found a quantitative meaure of "explosivity"; over the first $100 \mathrm{~s} \sim 2-6 \times 10^{9} \mathrm{~kg} / \mathrm{s}$ were ejected. The blast style of eruptions is indeed distinguished by extremely high mass ejection rates. Greater than $20 \%$ of the total erupted products of May 18, 1980, were ejected in less than $2 \mathrm{~min}$ of the blast. Much of the mass was concentrated into even shorter pulses at the peaks of the mass ejection events. Further quantification of both the eruptive style and the potential hazard is possible by noting that the "violence," that is, momentum [Walker, 1980], of the eruption is directly measured by the seismic force $F$ to within a factor of $\left(1+1 / \mathcal{M}^{2}\right)^{-1}$ (see equation 17). We propose an index of violence $M_{v}$ defined as

$$
M_{v} \equiv \frac{1}{2} \log \left|F_{\max }\right|,
$$

where $F_{\max }$ is the peak value of the seismic force in Newtons. The factor of $1 / 2$ is included to produce values comparable to standard volcanic indices and earthquake magnitude scales. For the Mount St. Helens blast $M_{v}=6.2$.

We have linked the blast process to its products, but the relationship has proved problematic. Is all of the blast deposit material from the laterally expanding, devasting blast or is a significant fraction of deposition from the accompanying ash cloud? Alternatively, we can interpret the results as addressing the final motivation-constraints on dynamical models. In 
this case, the important problem raised by this study concerns the mechanism by which the blast was directed. Why was it lateral? These questions pose challenges for future research and direct our understanding of the mass ejection process. Furthermore, the formulation presented here is generally applicable to any rapid, explosive volcanic eruption with significant jetting and can be used to study dome collapses and strombolian events as well as directed blasts.

\section{Appendix A: Equilibrium Flow Conditions}

If the gas and particle phases initially travel at different velocities, the relaxation time $\tau_{v}$ necessary for the particles to be accelerated to the gas velocity can be derived by balancing the particle acceleration against the Stokes drag. The standard result is

$$
\tau_{v}=\frac{\rho_{p} D^{2}}{18 \eta}
$$

where $\rho_{p}$ is the density of the particles, $D$ is the diameter of the particles, and $\eta$ is the gas viscosity corrected for the presence of particles [Rudinger, 1980]. This formulation of $\tau_{v}$ overestimates the relaxation time for high Reynolds number flows and therefore provides a conservative estimate of the time for equilibration. Thermal equilibrium is obtained over roughly the same timescale [Rudinger, 1980]. Appropriate values for $\rho_{p}$ and $\eta$ are $2000 \mathrm{~kg} / \mathrm{m}^{3}$ [Hoblitt and Harmons, 1993] and $8 \times 10^{-5} \mathrm{~Pa} \mathrm{~s}$ [Rudinger, 1980; Sengers and Watson, 1986]. The median particle size in the blast deposit is 0.1-1 mm [Moore and Sisson, 1981]. Equation (A1) implies that under the above conditions all particles 1 $\mathrm{mm}$ in diameter or less are in equilibrium with the gas phase after about $1.4 \mathrm{~s}$. This value of $\tau_{v}$ must be compared to the characteristic transit time in order to establish whether or not equilibrium occcurs.

Mass balance considerations require the fragmentation wave to travel into the initially stationary cryptodome at a velocity $v_{f}$ such that

$$
v_{f}=-v_{1} \frac{\rho_{1}}{\rho_{2}-\rho_{1}}
$$

where $\rho_{1}$ and $\rho_{2}$ are the densities behind and in front of the fragmentation wave, respectively, and $v_{1}$ is the average velocity of the flow behind the fragmentation wave. Therefore the time necessary for a particle to travel from the fragmentation wave front to the throat at velocity $v_{1}$ scales as $t \rho_{1} /\left(\rho_{2}-\rho_{1}\right)$ where $t$ is the time since the fragmentation wave left the throat. The maximum value of $\xi_{*}$ considered in this work is 0.5 which correponds to $\rho_{1} / \rho_{2}=0.5$ for $\phi=1$. Therefore the transit time for a particle is at most $t$. We assume that the fragmentation wave starts from the throat at the beginning of the lid removal process. After the first $1.4 \mathrm{~s}$ of a pulse, the dusty gas is in equilbrium if the solid volume fraction is large. In this case, equilibrium flow is a justified simplification for most $(>90 \%)$ of the pulsation process.

A dilute flow $\left(\xi_{*} \rightarrow 0\right)$ can have an arbirtrarily short transit time and may not reach equilbrium by the throat. In this case, it is useful to consider the situation with no coupling between the gas and the solid phases as a alternative end-member behavior. The exit velocity used in the momentum balance is the solid particle velocity and it must be estimated by a ballistic calculation. If the gas and solid phases are uncoupled, drag is negligible and the ballistic velocity $v_{b}$ is

$$
v_{b}=\sqrt{2 g h},
$$

where $g$ is the gravitational acceleration and $h$ is the eruption height above the vent. In order to conservativly estimate $\dot{M}$, we only consider the maximum value of $v_{b}$ corresponding to the maximum projectile height. The initial blast cloud was observed to rise to a maximum height of 5 or $6 \mathrm{~km}$ above the vent [Sparks et al., 1986]. Therefore the maximum ballistic exit velocity is between 310 and $340 \mathrm{~m} / \mathrm{s}$. The jet pressure is atmospheric since it is unlikely that a pure gas phase would reach the supersonic velocities. The equivalent expression to (19) in SI units is

$$
\frac{-F}{310} \geq \dot{M} \geq \frac{-F}{340} .
$$

These values are within the range considered in (19), therefore no separate calculations are required.

\section{Appendix B: Estimating the Mach Number}

We can estimate the Mach number for a steady expanding isentropic dusty gas by first considering the conservation of momentum [Rudinger, 1980]. As the fluid flows in the $x$ direction,

$$
\rho u \frac{d u}{d x}=-\frac{d P}{d x},
$$

where $u$ is the velocity of the flow and $P$ is the pressure. Eliminating the differential $d x$ from (B1) results in an expression for $d P / d u$. A fluid expanding isentropically has its pressure related to its density by the sound speed. Rearranging (B1) and substituting the sound velocity $c^{2} \equiv(\partial P / \partial \rho)_{s}$,

$$
\frac{-u}{c^{2}} d u=1 / \rho d \rho .
$$

If the sound speed were constant, the integration of (B2) would be trivial. However, a dusty gas generally has a variable solid volume fraction $\xi$ as it expands. We substitute (10) into (B2) and integrate from the throat to the vent to find a general expression for variable $\xi$ in the limit of the specific heat ratio $\Gamma \rightarrow 1$.

$\frac{1}{2}\left(1-\mathcal{M}_{*}^{2}\right)=\frac{\left(1-\xi_{*}\right)^{2}}{1-\xi}-\left(1-\xi_{*}\right)+\left(1-\xi_{*}\right)^{2} \ln \frac{\rho}{\rho_{*}} \frac{1-\xi_{*}}{1-\xi}$ 
The modified Mach number $\mathcal{M}_{*}$ denotes the velocity at the vent $u$ divided by the sound velocity at the throat $c_{*}$. We can eliminate the density ratio $\rho / \rho_{*}$ by noting that mass is conserved throughout the flow,

$$
\frac{\rho}{\rho_{*}}=\frac{u_{*} A *}{u A}=\frac{A_{*}}{A \mathcal{M}_{*}} .
$$

Similarly, since no solid magmatic material transforms to the volatile phase or vice versa, the solid phase is also conserved

$$
\frac{\xi}{\xi_{*}}=\frac{u_{*} A_{*}}{u A}=\frac{A_{*}}{A \mathcal{M}_{*}} .
$$

Combining (B3), (B4) and (B5),

$\frac{1}{2}\left(1-\mathcal{M}_{*}^{2}\right)=\frac{\left(1-\xi_{*}\right)^{2}}{1-\xi_{*} \frac{A_{*}}{A \mathcal{M}_{*}}}-\left(1-\xi_{*}\right)+\left(1-\xi_{*}\right)^{2} \ln \frac{1-\xi_{*}}{\frac{A \mathcal{M}_{*}}{A_{*}}-\xi_{*}}$.

Given a volume fraction $\xi_{*}$ and expansion ratio $A / A_{*}$, (B6) can be solved numerically for $\mathcal{M}_{*}$. The modified Mach number $\mathcal{M}_{*}$ is related to $\mathcal{M}$ by the ratio of the sound speeds of the vent and the throat. In the limit of $\Gamma \rightarrow 1$ the fluid expansion is isothermal and the sound speed varies only due to the variations in solid volume fraction,

$$
\mathcal{M}=\mathcal{M}_{*} \frac{1-\xi}{1-\xi_{*}}=\frac{\mathcal{M}_{*}-\xi_{*} \frac{A}{A_{*}}}{1-\xi_{*}}
$$

Equations (B6) and (B7) together provide a complete method of estimating the Mach number for particular values of $\xi_{*}$ and $A / A_{*}$. In the limit of negligible solid volume fraction $\left(\xi_{*} \rightarrow 0\right)$, the sound speed remains constant and the Mach number can be calculated numerically with the simplified equation

$$
\frac{A}{A_{*}} \mathcal{M}=\exp \left(\frac{\mathcal{M}^{2}-1}{2}\right)
$$

Acknowledgments. Correspondence and conversations with S. Kieffer, S. Malone, R. Waitt and R. Hoblitt have been valuable in understanding the geological and observational records. This paper is based on work supported under the National Science Foundation grant EAR-9903085 and is contribution 8678 of the Division of Geological and Planetary Sciences, California Institute of Technology.

\section{References}

Burger, R., and C. Langston, Source mechanism of the May 18,1980 , Mount St. Helens eruption from regional surface waves, J. Geophys. Res., 90, 7653-7664, 1985.

Christiansen, R. L., and D. W. Peterson, Chronology of the 1980 eruptive activity, U.S. Geol. Surv. Prof. Pap., 1250, 17-30, 1981.

Criswell, C. W., Chronology and pyroclastic stratigraphy of the May 18, 1980, eruption of Mount St. Helens, $J$. Geophys. Res., 92, 10237-10266, 1987.

Eichelberger, J. C., and D. B. Hayes, Magmatic model for the Mount St. Helens blast of May 18, 1980, J. Geophys. Res., 87, 7727-7738, 1982.
Friedman, J. D., G. R. Olhoeft, G. R. Johnson, and D. Frank, Heat content and thermal energy of the June dacite dome in relation to total energy yield, MayOctober 1980, U.S. Geol. Surv. Prof. Pap., 1250, 557567, 1981.

Glicken, H.. Rockslide-debris avalanche of the May 18, 1980, Mount St. Helens Volcano, Washington, U.S. Geol. Surv. Open-file Rep., 96-677, 1996.

Gorshkov, G. S., Directed volcanic blasts, Bull. Volcan., 26, 83-87, 1963.

Hickson, C., The May 18, 1980, eruption of the Mount St. Helens, Washington state: A synopsis of events and review of Phase I from an eyewitness perspective, Geosci. Can., 17, 127-131, 1990.

Hoblitt, R. P., Reply by R.P. Hoblitt to comment by M. Rosi on "May 18, 1980 Mt. St. Helens deposits in South Coldwater Creek, Washington", by R. V. Fisher, H. Z. Glicken, and R. P. Hoblitt, Bull. Volcanol., 52, 71-73, 1989.

Hoblitt, R. P., and R. S. Harmons, Bimodal density distribution of cryptodome dacite from the 1980 eruption of Mount St. Helens, Washington, Bull. Volcanol., 55, 421437, 1993.

Hoblitt, R. P., and C. D. Miller, Comment on Walker \& McBroome (1983), Geology, 12, 692-693, 1984.

Hoblitt, R. P., C. D. Miller, and J. W. Vallance, Origin and stratigraphy of the deposit produced by the May 18 directed blast, U.S. Geol. Surv. Prof. Pap., 1250, 401420, 1981.

Kanamori, H., and J. W. Given, Analysis of long-period seismic waves excited by the May 18, 1980, eruption of Mount St. Helens - a terrestrial monopole?, J. Geophys. Res., 87, 5422-5432, 1982.

Kanamori, H., J. W. Given, and T. Lay, Analysis of seismic body waves excited by the Mount St. Helens eruption of May 18, 1980, J. Geophys. Res., 89, 1856-1866, 1984.

Kieffer, S. W., Fluid dynamics of the May 18 blast at Mount St. Helens, U.S. Geol. Surv. Prof. Pap., 1250, 379-400, 1981.

Kieffer, S. W., and B. Sturtevant, Laboratory studies of volcanic jets, J. Geophys. Res., 89, 8253-8268, 1984.

Kieffer, S. W., and B. Sturtevant, Erosional furrows formed during the lateral blast at Mount St. Helens, May 18, 1980, J. Geophys. Res., 93, 14793-14816, 1988.

LaCroix, A., Remarques sur les materiaux de projection des volcans et sur la genese des roches pyroclastiques qu'ils constituent, Centenaire de la Societe Geologique de Francais, 2, 431-472, 1930.

Lipman, P. W., Geologic map of deposits and features of 1980 eruptions of Mount St. Helens, Washington, U.S. Geol. Surv. Prof. Pap., 1250, plate 1, 1981.

Marble, F. E., Dynamics of dusty gases, Ann. Rev. Fluid Mech., 2, 397-446, 1970.

Moore, J. G., and C. J. Rice, Explosive Volcanism: Inception, Evolution and Hazards, chap. Chronology and Character of the May 18, 1980, Explosive Eruptions of Mount St. Helens, pp. 133-142, National Academy Press, Washington, D.C., 1984.

Moore, J. G., and T. W. Sisson, Deposits and effects of the May 18 pyroclastic surge, U.S. Geol. Surv. Prof. Pap., 1250, 421-438, 1981.

Newhall, C., and S. Self, The volcanic explosivity index (VEI): An estimate of explosive magnitude for historical volcanism, J. Geophys. Res., 87, 1231-1238, 1982.

Nielsen, E., R. Waitt, and S. Malone, Eyewitness accounts and photographs of the 18 may 1980 eruption of Mount St. Helens, Wash., New Mexico Bureau of Mines and Mineral Resources, Bulletin, 131, 203, 1989.

Nishimura, T., Source parameters of the volcanic eruption 
earthquakes at Mount Takachi, Hokkaido, Japan, and a magma ascending model, J. Geophys. Res., 100, 12,46512,474, 1995.

Rosenbaum, J. G., and R. B. Waitt, Summary of eyewitness accounts of the May 18 eruption, U.S. Geol. Surv. Prof. Pap., 1250, 53-67, 1981.

Rudinger, G., Fundamentals of Gas-Particle Flow, Elsevier Sci., New York, 1980.

Sarna-Wojicki, A., A. Shipley, R. B. Waitt, D. Dzurisin, and S. H. Wood, Areal distribution, thickness, mass, volume, and grain size of air-fall ash from the six major eruptions of 1980, U.S. Geol. Surv. Prof. Pap., 1250, 577-600, 1981.

Scandone, R., and S. D. Malone, Magma supply, magma discharge and readjustments of the feeding system of Mount St. Helens during 1980, J. Volcanol. Geotherm. Res., 23, 239-262, 1985.

Sengers, J., and J. Watson, Improved international formulations for the viscosity and thermal-conductivity of water substance, J. of Phys. Chem. Ref. Data, 15(4), 1291$1314,1986$.

Sisson, T. W., Blast ashfall deposit of May 18, 1980 at Mount St-Helens, Washington, J. Volcanol. Geotherm. Res., 66, 203-216, 1995.

Sparks, R. S. J., J. G. Moore, and C. J. Rice, The initial giant umbrella cloud of the May 18th, 1980, explosive eruption of Mount St. Helens, J. Volcanol. Geotherm. Res., $28,257-274,1986$.

Sparks, R. S. J., M. I. Bursik, S. N. Carey, J. Gilbert, L. Glaze, H. Sigurdsson, and A. W. Woods, Volcanic Plumes, John Wiley, New York, 1997.

Thompson, P. A., Compressible-Fluid Dynamics, McGrawHill, New York, 1972.

Uhira, K., and M. Takeo, The source of explosive eruptions of Sakurajima Volcano, Japan, J. Geophys. Res., 99, 17,775-17,789, 1994.

Uhira, K., H. Yamasato, and M. Takeo, Source mechanism of seismic waves excited by pyroclastic flows observed at Un- zen Volcano, Japan, J. Geophys. Res., 99, 17,757-17,773, 1994.

Voight, B., Time scale for the first moments of the May 18 eruption, U.S. Geol. Surv. Prof. Pap., 1250, 69-86, 1981.

Waitt, R. B., Comment on Walker and McBroome (1983), Geology, 12, 693, 1984.

Waitt, R. B., and D. Dzurisin, Proximal air-all deposits from the May 18 eruption: Stratigraphy and field sedimentology, U.S. Geol. Surv. Prof. Pap., 1250, 601-616, 1981.

Walker, G., The Taupo pumice: Produce of the most powerful known (ultraplinian) eruption?, J. Volcanol. Geotherm. Res., 8, 69-94, 1980.

Walker, G. P. L., and L. A. McBroome, Mount St. Helens 1980 and Mount Pelee 1902: flow or surge?, Geology, 11, 571-574, 1983.

Wilson, L., R. S. J. Sparks, T. C. Huang, and N. D. Watkins, The control of volcanic column heights by eruption energetics and dynamics, J. Geophys. Res., 83, 1829-1836, 1978.

Wilson, L., R. S. J. Sparks, and G. P. L. Walker, Explosive volcanic eruptions; IV, the control of magma properties and conduit geometry on eruption column behaviour, Geophys. J. R. Astr. Soc., 63, 117-148, 1980.

E. E. Brodsky and H. Kanamori, Seismological Laboratory, MC 252-21, California Institute of Technology, Pasadena, CA 91125. (brodsky@gps.caltech.edu; hiroo@gps.caltech.edu)

B. Sturtevant, Graduate Aeronautical Laboratories, California Institute of Technology, Pasadena, CA 91125. (brad@galcit.caltech.edu)

(Received January 5, 1999; revised July 22, 1999; accepted September 7, 1999.) 